\title{
A Clinical Trial Based on Reward Contingency to Improve Prone Tolerance and Motor Development is Feasible in 3- to 6-Month-Old Infants
}

\author{
Tanya Tripathi \\ Stacey C. Dusing \\ Ohio State University University of Southern California \\ Peter E. Pidcoe, Yaoying Xu, Mary S. Shall, and \\ Daniel L. Riddle \\ Virginia Commonwealth University
}

\begin{abstract}
Aims: The American Academy of Pediatrics recommends "parents to incorporate supervised, awake 'prone play' in their infant's routine to support motor development and minimize the risk of plagiocephaly". The purpose of this feasibility study was to compare usual care to a reward contingency-based intervention, developed to increase prone tolerance and improve motor skills. Methods: Ten full-term infants, 3-6- months old, with poor prone tolerance were randomized to either the Education group or Reward contingency group. Each group participated in three parent education sessions and 15 intervention sessions, over the period of three weeks. Infants in the Reward contingency group used the Prone Play Activity Center, a technology developed to reinforce motor behavior of infants in prone position. Intervention frequency and parent feedback data determined the feasibility of the interventions. Results: Infants in the Reward contingency group practiced a median of 12 of the 15 anticipated intervention sessions in the Prone Play Activity Center. These infants used the device for a mean of 18 minutes per day. Parents of infants in the Education group practiced a median of 10 sessions of the 15 anticipated intervention sessions. Conclusion: The reward contingencybased intervention is feasible for use in a future clinical trial with some modifications.
\end{abstract}

Keywords: motor control, motor learning, prone positioning, rehabilitation technology

\footnotetext{
Tripathi is with the Pediatric and Rehabilitation Laboratory, Ohio State University, Columbus, OH, USA. Dusing is with the Division of Biokinesiology and Physical Therapy, University of Southern California, Los Angeles, CA, USA. Pidcoe, Shall, and Riddle are with the Department of Physical Therapy; Xu is with the Department of Counselling and Special Education; Virginia Commonwealth University, Richmond, VA, USA. Tripathi (Tanya.Tripathi@osumc.edu) is corresponding author.
} 
The American Academy of Pediatrics recommends "parents and caregivers to incorporate supervised, awake 'prone play' in their infant's routine to support motor development and minimize the risk of head deformities such as positional plagiocephaly" ("Back to sleep, tummy to play", 2008; Chizawsky \& ScottFindlay, 2005). However, approximately $70 \%$ of 4- to 5-month-old infants spend more time in supine or supported sitting compared to prone position during the day (Dudek-Shriber \& Zelazny, 2007). Recent estimates suggest that in the year of 2013, 20\% of $\sim 4$ million infants in the USA experienced some degree of positional skull deformation (Klimo et al., 2016). Excessive supine lying and limited prone play in infants' routine are key factors associated with the risk of positional plagiocephaly (Collett, Breiger, King, Cunningham, \& Speltz, 2005; van Vlimmeren et al., 2007).

Infants' poor tolerance for prone and parents' hesitation towards prone play likely leads to minimum practice of motor skills in the prone position (Zachry \& Kitzmann, 2011). Studies have suggested that strength and coordination, gained by the practice of raising the upper body and holding the head while in prone, develops prone motor skills and reduces the risk of plagiocephaly in infants (Bartlett \& Fanning, 2003; Hunter \& Malloy, 2002). Current approaches to improve infants' tolerance for prone play include educating parents through brochures and using commercially available prone positional supports such as u-shaped pillows and play gyms with toys. Educational strategies alone do not appear to be effective in helping parents implement an effective prone play program (Koren, Reece, Kahn-D'angelo, \& Medeiros, 2010). In addition, toys commonly used for prone play are easily accessible from other positions such as supine or side lying. For instance, commercial play gyms do not provide a contingent response to the infants' movements in prone, limiting their ability to motivate the infant to play in this position.

Interventions that are effective at promoting motor skills in infants have some common characteristics, such as (1) they encourage child-initiated movements, (2) they allow trial and error to support motor learning, and (3) they educate parents on the importance of movement variability and environment enrichment (Dusing et al., 2018; Morgan et al., 2016; Novak et al., 2013). The proposed intervention blends these characteristics with technology in an attempt to improve infants' tolerance for prone and to support prone motor skills. Our intervention is also in line with the concept of Vygotsky's Zone of Proximal Development which is described as "the distance between the actual and the potential level of development". The skill of independent problem solving directs the level of actual development; however, the level of potential development is determined through problem solving under adult guidance or in collaboration with more capable peers (Vygotsky, 1978). Thus, through appropriate guidance and feedback, development can be "boosted" in infants and children. The proposed intervention uses the concept of Vygotsky's Zone of Proximal Development by practicing the key elements of (1) positive reinforcement and (2) "just right challenge", to scaffold the motor development of infants at 3 to 6 months of age. The element of positive reinforcement is derived from the principle of reward contingency. A contingency is defined as "a temporal relationship between two events, where the presence or use of one event increases or decreases the likelihood of the occurrence of the other one" (Dunst, Trivette, Raab, \& Masiello, 2008). According to the principle of 
reward contingency, a behavior can be encouraged by associating it with positive reinforcements or rewards, a strategy commonly used by school teachers to increase academic engagement in school aged children (Scott, 2001; Thorne \& Kamps, 2008). Multiple studies have found that infants as young as 3 months of age can change their behavior when a reward is introduced contingent to motor activity (Dunst et al., 2008; Enright, Rovee-Collier, Fagen, \& Caniglia, 1983; Gerhardstein, Kraebel, \& Tse, 2006; Heathcock, Bhat, Lobo, \& Galloway, 2004; Rovee-Collier \& Gekoski, 1979; Sargent, Reimann, Kubo, \& Fetters, 2015; Thelen, 1995; Tripathi et al., 2019). In our previous research, about $50 \%$ of 3- to 6-month-old, typically developing infants discovered the relationship between their upper body movements (including head) in prone and the activation of a toy. These infants learned the activation of a toy that contingently responded when the infant lifted their head over a threshold (Tripathi et al., 2019). The second principle of the intervention is to challenge the infant just beyond their current ability, reward success, and gradually increase the challenge, which we refer to as the "just right challenge" (Dusing et al., 2018, 2019; Harbourne et al., 2018).

The primary purpose of this study was to assess the feasibility of administering two home-based interventions for a future randomized clinical trial. These interventions were developed to increase the tolerance for prone position and improve motor skills in 3- to 6-month-old infants. Specifically, the objectives were to determine the feasibility of coaching parents to deliver the proposed interventions, evaluate parent's ability to implement the interventions independently and evaluate the feasibility of scoring two measures of motor skills and a test of prone tolerance for use in future research studies.

\section{Methods}

\section{Participants and Setting}

Participating infant/parent dyads self-identified after hearing about the research study from community flyers and letters. Eligible infants were between 3 to 6 months of age and had poor tolerance and motor skills in the prone position. Poor prone tolerance was identified from parent reports followed by an in-person screening of the same to confirm inclusion in the study. Fussing/crying for $>30$ seconds during a five-minute period in prone confirmed an infant's intolerance for prone play. Poor prone motor skills were defined as a score between 2 to 4 in the prone subsection of the Alberta Infant Motor Scale (Piper, Pinnell, Darrah, Maguire, \& Byrne, 1992). At the time of enrollment, all infants were able to raise their head asymmetrically or symmetrically to a minimum of 45 degrees but could not pushup on extended arms in prone or move out of prone position. Infants born with brain injury or any neurological event associated with a risk of neurodevelopmental disabilities, musculoskeletal deformity, genetic syndromes, visual and hearing problems, or any other medical complications limiting participation were excluded from the study.

Thirteen infants were assessed for enrollment after the parents reported their infant's poor tolerance in prone position. Of these infants, 10 infants were eligible (40\% female; $M_{\text {age }}=4.19$ months, $S D=0.7$ months) and were enrolled in the study. Consistent with the Consolidated Standards of Reporting Trials guidelines 
for feasibility trials (Eldridge et al., 2016), the sample size was determined to address our purpose of evaluating the feasibility of the proposed intervention and not to conduct inferential statistics. Parents provided informed consent for their infant prior to participation.

All infants participated in the same assessment schedule, regardless of the group assignment. Parents signed informed consent for their infant's participation and filled out their child's demographic information. Following baseline assessment, infants were randomized using a computer-generated random sequence to the Reward contingency group or the Education group. All assessments and intervention sessions were video recorded and conducted in the infant's home. The same researcher (T.T.) conducted the assessments and intervention sessions; however, the assessments were scored/coded from a video by a trained and reliable data coder blinded to the group assignment.

\section{Intervention}

Education Session for Both Groups. At baseline, day 7, and day 14 of the study, all parents participated in a session on the importance of including prone play in an infant's routine. The education session reviewed information parents might receive on "tummy time" at their physician's office or on the internet, thus controlling for parents' knowledge on the benefits of prone play between the two groups. All parents received the "Back to sleep, Tummy to Play" brochure that included tips to encourage prone play such as using toys, a towel roll, or providing tummy time on the caregiver's chest (Figure 1). Parents were asked to incorporate at least 30 minutes (in four to five short periods initially) of prone play in their infant's daily routine for 15 days over a period of three weeks. They had the option of pacing the intervention days based on their schedule and availability. During this session, parents also received an intervention manual of the same information provided by the researcher verbally.

Reward Contingency Technology. In addition to the education session, the Reward contingency group received the Prone Play Activity Center (PPAC) on loan

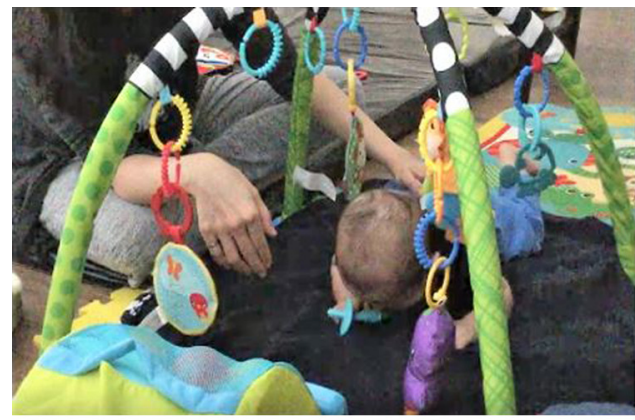

A

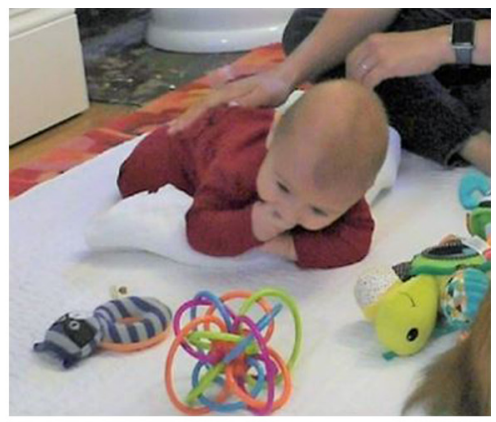

B

Figure 1 - Strategies for prone play. Panel A shows an image of a commercial play gym used during a parent education session. Panel B shows a towel roll and set of toys used to encourage prone play during a parent education session. 
for three weeks. To include prone play in the infant's routine, parents were instructed to use the PPAC for a total of 30 minutes per day for 15 days over three weeks (Figure 2). The parents had access to use the PPAC for the whole intervention period, whenever they wanted within their home. The PPAC is a device developed by the research team to provide a reward contingent to the infant raising his/her head to a certain height. A variety of toys were connected to the PPAC as the source of reward activation, but in all cases the toy moved, played music, and/or lighted up. The PPAC had the following components: 1) ultrasonic sensors, 2) Arduino Uno microcontroller, and 3) moving/singing toy. The ultrasonic sensors calculated the position of the infant's head from the floor. These sensors record distance based on the time required for the high-frequency sound waves, emitted from the sensors, to reflect from an object (infant's head) (Griffith \& Biswas, 2017). The Arduino Uno microcontroller compared the infant's head height to a preset parameter or height of a threshold and activated the toy if conditions were met. To ensure the infants received continuous reinforcement in response to their head raised in prone, the PPAC activated the toy when the infant raised his/her head at or above the height of the threshold and turned the toy off only when the infant's head height was below the threshold.

Reward Contingency Intervention. After the education session, the researcher demonstrated the functions of the PPAC and coached parents to set it up independently. Infant's average head height, calculated by the PPAC at baseline, was used to set three levels of difficulty: easy, moderate, and challenging. These levels were used to progress the degree of challenge as the infant learned to activate the toy and increased prone tolerance. The easy level was set at $25 \%$ below the average head height. At this threshold height, infants in our pilot work activated the

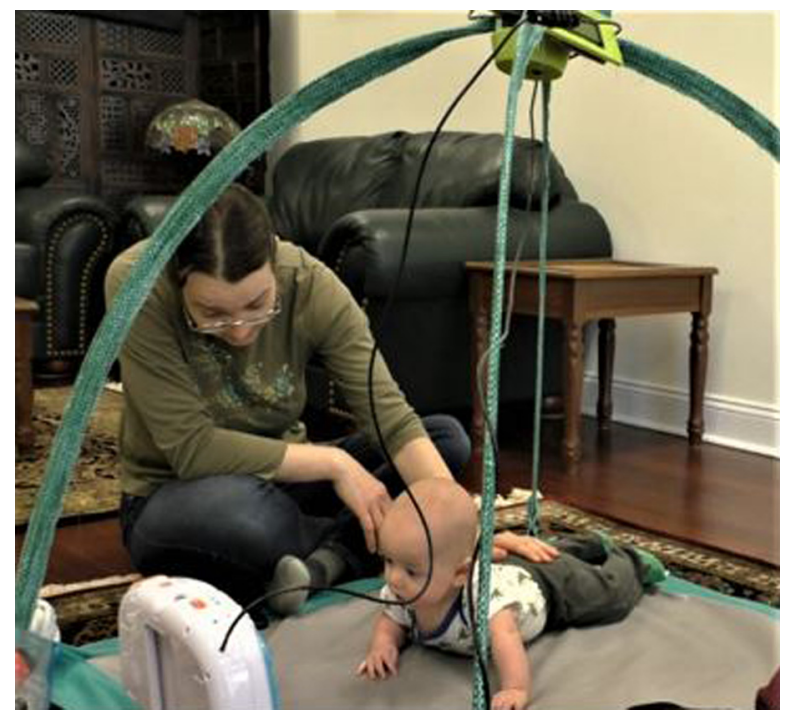

Figure 2 - Prone Play Activity Center. The image is of a parent and infant in the Reward contingency group using the Prone Play Activity Center. 
PPAC toy easily by raising their head in prone with or without bearing weight on the forearms. The moderate threshold height was set at the infant's average head height at baseline. For the challenging level the threshold height was set at $25 \%$ above the average head height. Parents were provided with three numerical values that corresponded to their infant's easy, moderate, and challenging level in the PPAC. All parents were asked to begin the intervention at the easy level to ensure the infants received the opportunity to experience the association between their head raise and toy activation multiple times in the learning phase. They were encouraged to progress from easy to moderate and then to the challenging level only when the infant tolerated prone play for 30 minutes at the previous level. By training parents to set the individualized threshold heights in the PPAC, the proposed intervention coached parents to determine what was "just right" for their infant based on their infant's changing prone motor abilities. During our pilot work, we observed some infants in the PPAC activate the toy throughout the intervention period without lowering their head, so no reactivation of the toy was needed. However, infants who held their head above the threshold and activated the toy also demonstrated an extended period of prone tolerance. Thus, the criteria to advance to a higher "just right" level or threshold height relied on prone tolerance as the primary metric while also accounting for the work required in activating the toy.

Parents were encouraged to be in view of the infant during the intervention but were asked not to directly engage with the infant. If the infant fussed or cried, parents were asked to gently pat the infant's back to soothe the infant, talk/sing to the infant, or move the infant out of prone if the calming strategies did not work. Parents had 24-hour access to the PPAC in the home and could pace the intervention in several short periods to avoid fatigue and gradually increase the duration based on the infant's behavioral state.

\section{Assessments of Feasibility}

The primary aim of this study was to determine the feasibility of administering the proposed interventions for comparison in future studies. In order to address our aim, the feasibility of enrollment in both the interventions and all assessments needed to be determined. To estimate the enrollment and retention rate, we tracked the number of parents who expressed interest in the study as well as the number of parents screened for eligibility, enrolled, and retained in the study. Intervention feasibility was determined by the degree to which the therapist and parent each adhered to the intervention.

Feasibility of Intervention. A checklist of the key components of both the interventions (see Supplementary Material 1 [available online]) were used to determine if the researcher followed the intervention procedures. After each intervention session, the researcher self-assessed her adherence to the intervention guidelines on the checklist. In addition, a second researcher (SCD) marked adherence on the same checklist from video recordings of $40 \%$ of the intervention sessions to measure interrater adherence.

Parent Adherence. All parents received an activity log to document the total duration of prone play per day for 15 days over three weeks (see Supplementary 
Material 2 [available online]). Parents could choose between a paper or electronic version of the activity $\log$. The activity $\log$ had 17 questions on infant's play positions and device use. Embedded within this questionnaire on infant activities, parents reported the amount of time the infant was in prone every day: $<15$ minutes, 15-30 minutes, and $>30$ minutes of prone time. For infants in the Reward contingency group, the activity log had additional questions specific to the PPAC; the number of minutes in the PPAC and the daily "just right" level used during prone play. A parent who advanced their infant's "just right" level or threshold height without the infant meeting the increment criteria of 30 minutes of prone play per day in the PPAC was considered incorrect in setting the threshold height in the PPAC. In addition, parents were asked to provide a yes/no response to indicate if their infant met the increment criteria to move to the moderate and challenging level of prone play. Any day that the log was not completed was recorded as missing data. If the log was completed with no record of time spent in prone (inside or outside of the PPAC for infants in the Reward contingency group), a " 0 " was included for the duration of prone on that day.

Feasibility of Outcome Measures. While previous literature has found an association between poor prone tolerance and motor outcomes (Hewitt, Stanley, \& Okely, 2017), quantification of prone tolerance is typically based on parent reports (Zachry \& Kitzmann, 2011). To quantify change in prone tolerance, we developed a measure of prone tolerance for infants in our study. All infants were tested for their prone tolerance at baseline and end of intervention. The infant was placed in prone by the researcher in front of a mirror toy for a maximum of 15 minutes. During the prone tolerance test, the researcher talked to the infant in a positive and encouraging tone. If the infant started to fuss and cry, a rattle was presented in front of the infant and the researcher would pat the infant's back to soothe the infant. The test ended after 15 minutes or stopped any time the infant cried (Brazelton infant behavioral state 6) continuously for $>30$ seconds (Brazelton $\&$ Nugent, 1995). The time lapse between the start and end of the trial was calculated as a measure of infant's tolerance for prone position. The score ranged from 0.5-15 minutes. A data coder blinded to group assignment scored all the videos to determine the prone tolerance, and a second data coder scored all of the same videos for reliability. The intraclass correlation coefficient (ICC) was calculated using a two-way mixed effects, absolute agreement, and multiple rater model (ICC $[3, \mathrm{k}]$ ) which demonstrated high inter-rater reliability (prone tolerance $\mathrm{ICC}=0.94)(\mathrm{Koo} \& \mathrm{Li}, 2016)$.

Two reliable and valid measures, the Alberta Infant Motor Scales (AIMS; Darrah, Barlett, Maguire, Avison, \& Lacaze-Masmonteil, 2014; Piper et al., 1992) and Gross Motor Function Measure-88 (GMFM-88; Russell, Rosenbaum, Wright, \& Avery, 2013), were administered and video was recorded at baseline and end of intervention. While the assessor was not blinded to group assignment, a standard protocol of placing the child in supine, prone, sitting, and standing was used for all children. The assessor was familiar to the child and family, which increased the likelihood of engagement and increased the validity of the assessment. Both motor assessments were scored from videos by a blinded, reliable data coder. Interrater reliability for the blinded coder was very high [AIMS ICC $(3, \mathrm{k})=0.99$ and GMFM-88 ICC $(3, \mathrm{k})=0.96]$. While GMFM-88 is designed for children with 
Cerebral palsy and Down syndrome, we used it for typically developing children because GMFM-88 samples a variety of motor skills that are "typical" of normal development from 0-5 years of age. (GMFM: Russell et al., 2013) It was important to determine the feasibility of completing both the AIMS and GMFM-88 to evaluate sensitivity of these measures to change in response to the Reward contingency intervention.

\section{Data Analysis}

Descriptive statistics were used to study the sample of participants and criteria to evaluate feasibility were determined a priori. Enrollment was considered feasible if $75 \%$ of the eligible infants were enrolled in the study. Retention was considered feasible if $90 \%$ of the enrolled infants completed the study. The feasibility of the researcher delivering the proposed interventions was determined by evaluating the percent of time the researcher reviewed $90 \%$ key principles of the intervention with the parents at baseline, day 7, and 14 of the study. The feasibility of the parent completing the intervention as designed was measured by the percent of time the parents completed 30 minutes of prone play on at least $85 \%$ of the planned session (in the PPAC for the Reward contingency group). The feasibility of the parent progressing the intervention as intended was evaluated by determining the percent of time the parents correctly identified the "just right" level of prone play at each visit. The measure of prone tolerance was considered feasible to use in the future efficacy study if more than $90 \%$ of the time infants completed the measure without achieving the lowest or highest score. The AIMS and GMFM- 88 were considered feasible if $90 \%$ of the time both assessments could be completed within a single visit.

\section{Results}

\section{Study Feasibility}

Enrollment and Retention Feasibility. Of the infants screened for eligibility, 76\% (10 of 13 infants screened after their parent reported poor prone tolerance on the phone) were eligible for participation. All infants who met the eligibility criteria were consented to participate and completed the baseline assessment. These 10 infants were randomized with five infants in each intervention group (Table 1). All five infants in the Education group completed the study. In the first week of the study, one infant in the Reward contingency group withdrew due to sibling interference with the PPAC and parental preference. A flow diagram of the progress of the participants through the phases of this parallel-randomized feasibility trial is presented in Figure 3.

Intervention Feasibility. The researcher completed 90\% (27/30) of the total required intervention sessions. Data of the child who withdrew from the Reward contingency group before the final visit was excluded. The researcher who conducted the education session for both the groups and guided parents on the use of PPAC in their infant's prone play routine, self-reported $100 \%$ adherence to the intervention. A 100\% interrater adherence to the intervention guidelines was found when a second researcher (S.C.D.) scored the primary researcher's (T.T.) ability to 


\section{Table 1 Participants Characteristics}

\begin{tabular}{lcc}
\hline & $\begin{array}{c}\text { Reward Contingency } \\
\text { Group }\end{array}$ & $\begin{array}{c}\text { Education } \\
\text { Group }\end{array}$ \\
\hline Age $M(S D)$ & $3.24(2.37)$ months & $4.1(0.49)$ months \\
Female & $40 \%(2)^{*}$ & $60 \%(3)^{*}$ \\
Not Hispanic or Latino & $75 \%(3)^{*}$ & $100 \%(5)^{*}$ \\
White & $100 \%(5)^{*}$ & $100 \%(5)^{*}$ \\
Primary caregiver age & & $60 \%(3)^{*}$ \\
26-35 years & $75 \%(3)^{*}$ & $40 \%(2)^{*}$ \\
$36-45$ years & $25 \%(1)^{*}$ & $80 \%(4)^{*}$ \\
Primary caregiver education level & & $20 \%(1)^{*}$ \\
$\quad$ Bachelor's & & \\
$\quad$ Master's & $100 \%(5)^{*}$ & $40 \%(2)^{*}$ \\
Primary caregiver employment status & & $60 \%(3)^{*}$ \\
$\quad$ Keeping house & $50 \%(2)^{*}$ & \\
Working full time & $50 \%(2)^{*}$ & \\
\hline
\end{tabular}

*The number in the parenthesis represents the number of infants.

follow the intervention guidelines. The interrater adherence was measured from the videos of both the interventions recorded at baseline, day 7, and day 14 .

Parent Adherence. Of the anticipated 15 days of the intervention, parents of infants in the Reward contingency group and Education group reported information on prone play in the activity log for a median of 12 days (range 5-15 days) and a median of 10 days (range 5-15 days), respectively. One parent in the Reward contingency group did not complete the log after the first week because the infant spent most of the intervention days in a daycare facility that did not track prone play time. On all the days the log was completed, parents in both groups reported prone play in their infant's routine. Parents of infants in the Reward contingency group reported their infant practiced 15-30 minutes and $>30$ minutes of cumulative prone play (in or out PPAC) for $38 \%$ and $45 \%$ of the time, respectively (Table 2). On $67 \%$ of the reported days, infants in the Education group practiced prone play for $15-30$ minutes and $17 \%$ of the time for $>30$ minutes per day (Table 2). Only $25 \%$ of the time, parents in the Reward contingency group used the PPAC for $>30$ minutes per day during the study. Each day, the PPAC was used for a duration of $M=18, S D=7$ minutes. All parents received credit for correctly identifying the "just right" level of prone play when asked during the weekly visits. However, 75\% (3/4) of the parents progressed their infant to a higher threshold level before their infant could tolerate prone play in the PPAC for $>30$ minutes at their current difficulty level. These parents reported the suggested level of threshold set to activate the toy was too easy for their infant. Yet, parents often reported that completing 30 minutes in the PPAC was difficult due to infants' poor tolerance for prone. Thus, parents increased the challenge to lift the head higher, rather than extending the duration, the opposite of what was in the intervention protocol, demonstrating limited adherence to the intervention protocol. 


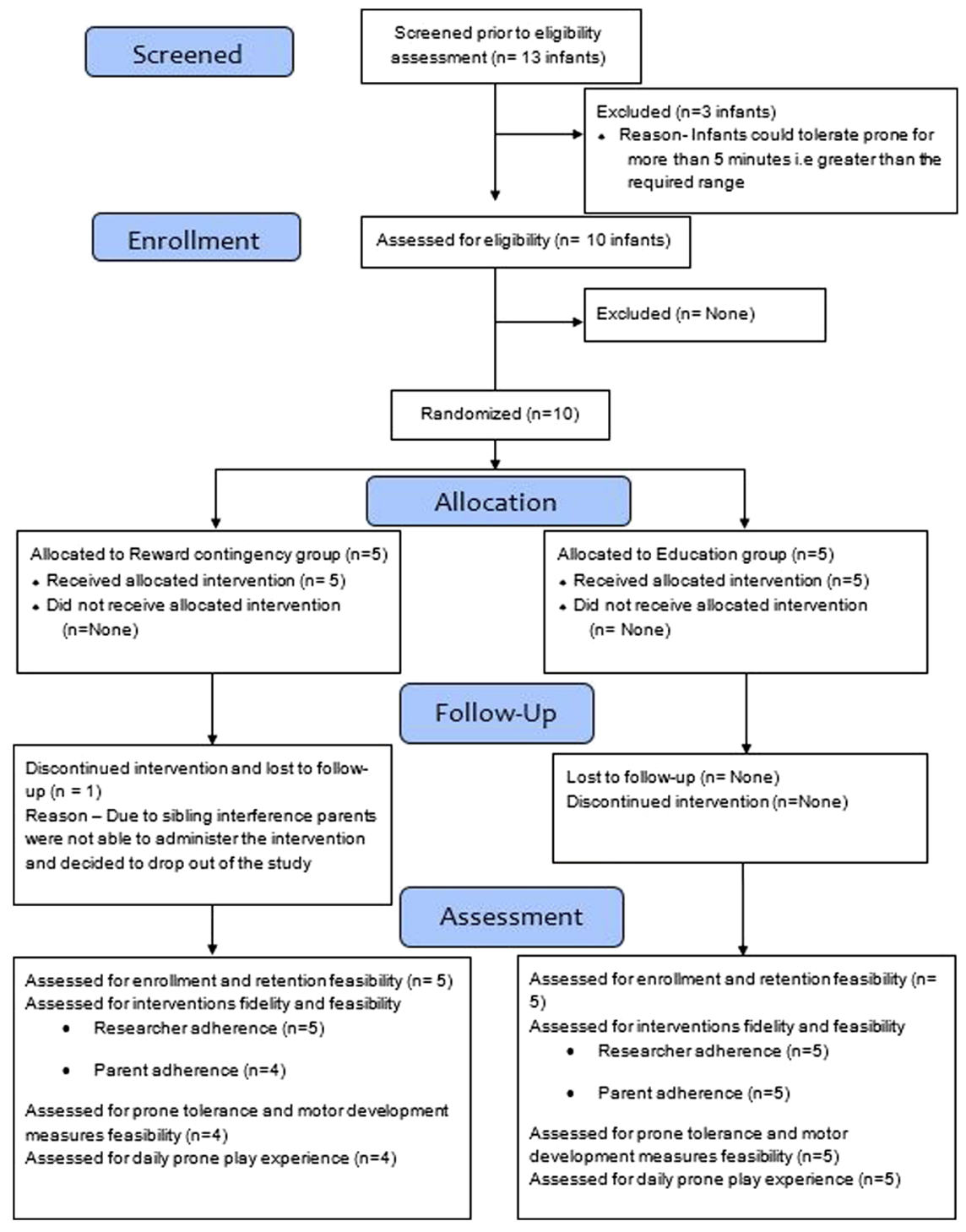

Figure 3 - Consolidated Standards of Reporting Trials flow diagram. This figure illustrates the progress of infants in the study through the feasibility trial.

Feasibility of the Outcome Measures. Within a single assessment visit, the research team was able to complete the tests of motor development (AIMS and GMFM-88) and prone tolerance on all the infants who participated in the study. In addition to this, $95 \%$ of the time infants completed the prone tolerance measure without achieving the lowest ( 0.5 minutes) or highest ( 15 minutes) score of the measure. 


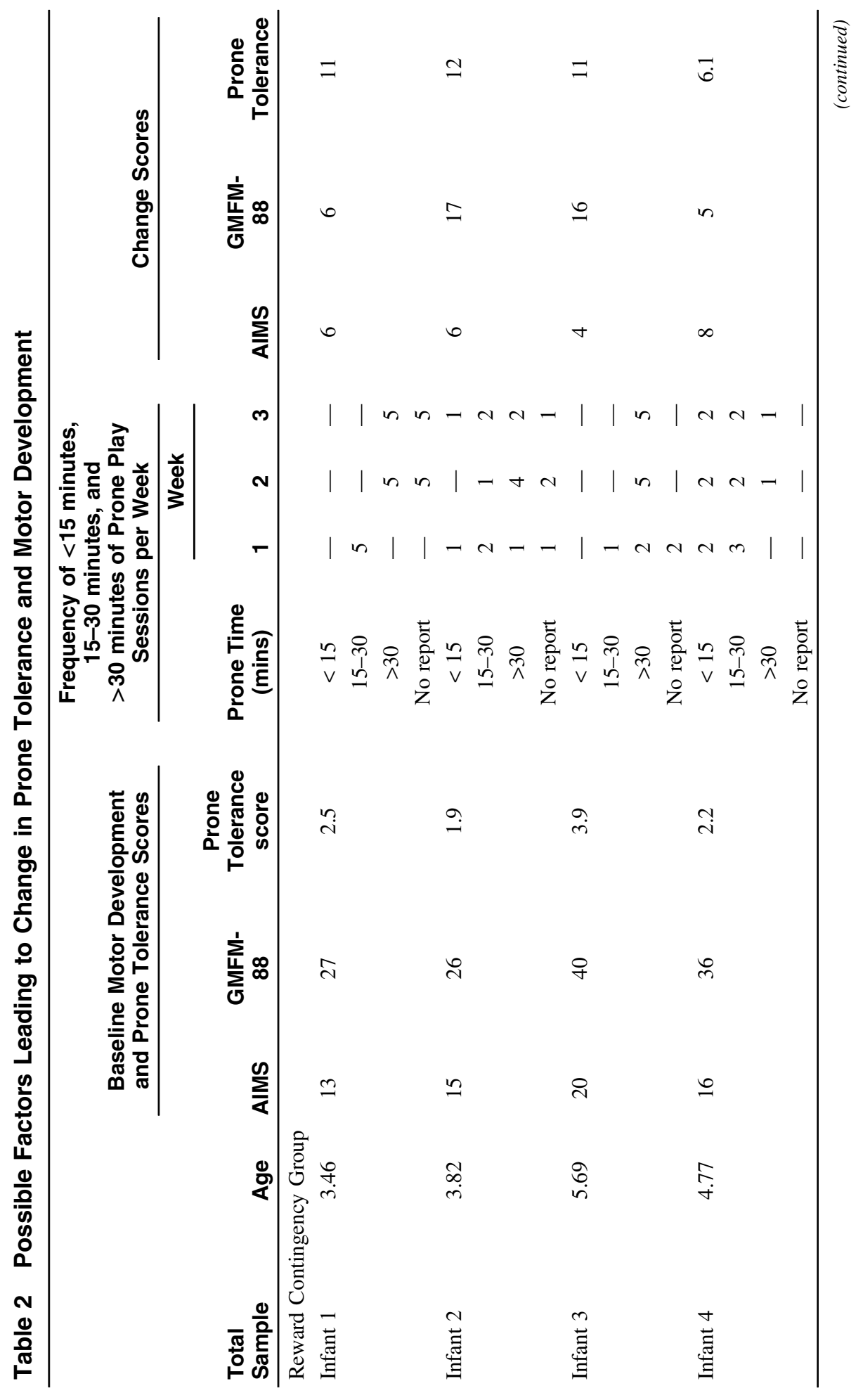




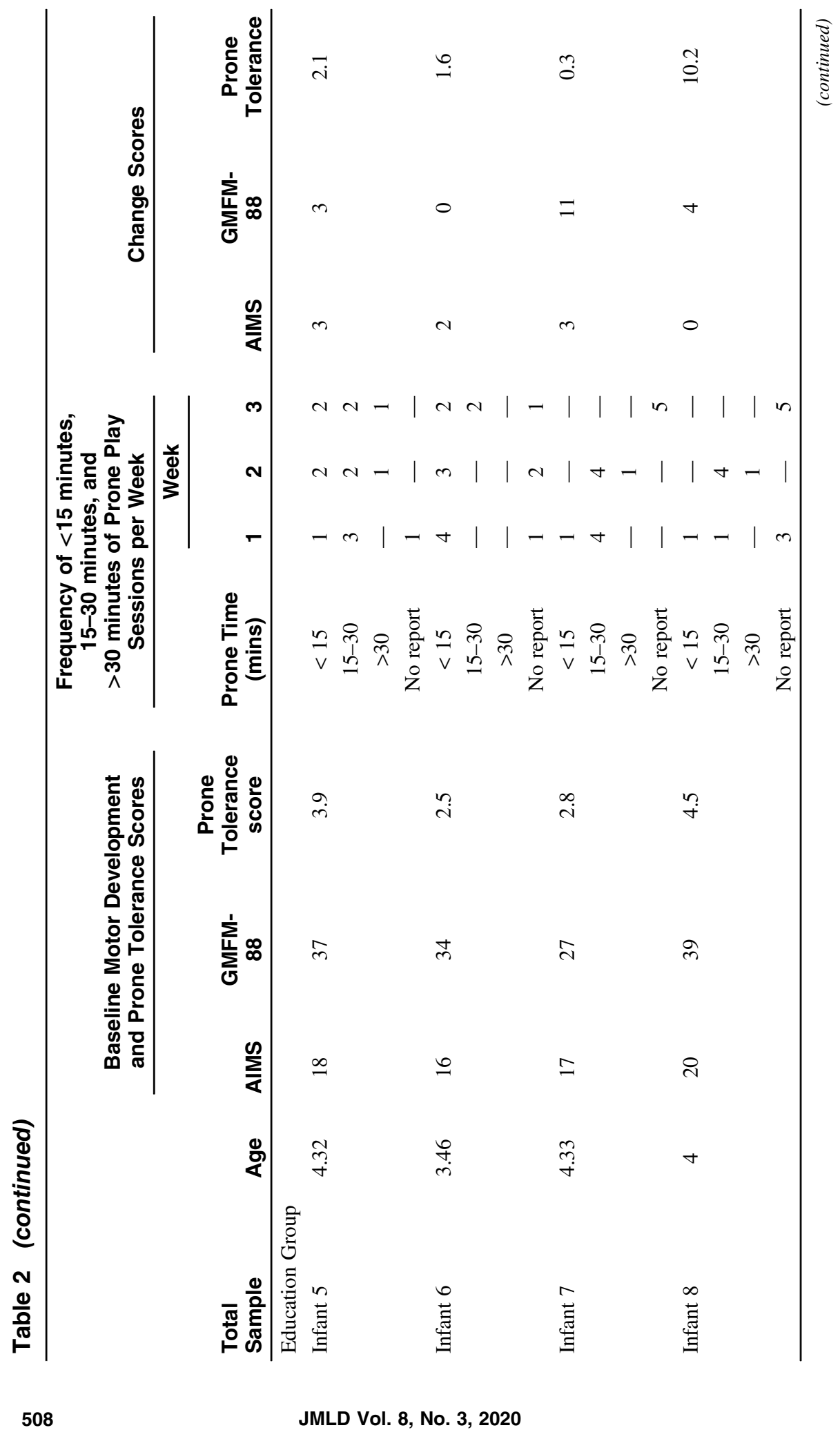




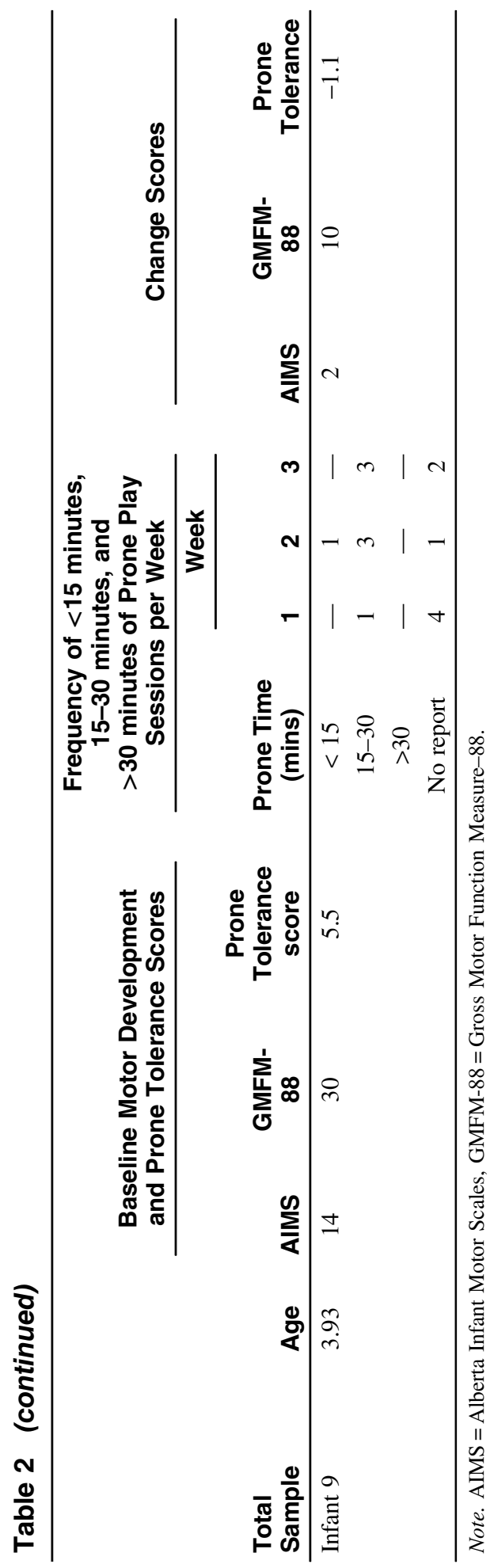




\section{Pilot Efficacy Data}

The purpose of this study was not to evaluate the efficacy of this intervention. However, the data provides a potential source of information for planning future studies. Although, the mean, standard deviation, and effect sizes are included to pilot the next efficacy trial, no group comparisons are provided as the study was not powered to evaluate the efficacy of the intervention. Change in the motor abilities and prone tolerance from baseline to end of intervention are reported in Table 2.

Prone Tolerance. The Reward contingency group had a mean change in their prone tolerance score from baseline to end of intervention of 10.0 minutes $(S D=2.72 ; 95 \%$ confidence interval [CI; 7.4, 12.7]; $n=4)$ while the Education group had a change of only 2.62 minutes $(S D=4.41 ; 95 \%$ CI $[-1.2,6.4] ; n=5)$. The effect size for the change in prone tolerance score from baseline to end of intervention was $1.91,95 \%$ CI $(0.72,2.92)$.

Motor Development. From baseline to end of intervention, infants in the Reward contingency group $(n=4)$ had a mean change of 6 points $(S D=1.63 ; 95 \%$ CI [4.4, 7.6]) in their AIMS raw score and infants in the Education group $(n=5)$ had a mean change of 2 points $(S D=1.22 ; 95 \%$ CI [0.93, 3.07]). In addition, the Reward contingency group had an average change of 11 points $(S D=6.38 ; 95 \%$ CI [4.7, 17.2]) in their GMFM-88 scores and infants in the Education group had an average change of 5.6 points $(S D=4.72 ; 95 \%$ CI $[1.4,9.7])$ from baseline to end of intervention. The effect size for the change in AIMS score was $1.42,95 \%$ CI $(0.39$, $2.46)$ and change in GMFM-88 score was $1.31,95 \%$ CI $(0.24,2.26)$.

\section{Discussion}

Our study suggests that it is feasible to administer an intervention developed using a reward contingency paradigm combined with parent education to enhance prone tolerance and motor development in 3- to 6-month-old infants. A clinical trial comparing the efficacy of the Reward contingency-based intervention to usual care is feasible in the future. However, some modifications in parents' training and methods for progressing the difficulty of the Reward contingency intervention is needed to achieve the targeted dose and intensity in the future studies.

\section{Intervention Feasibility and Parent Adherence}

All parents in the study demonstrated understanding of the prone play recommendations provided to both the groups. In addition, parents independently implemented the intervention at each of the home visits. However, they had difficulty in practicing the recommended dose of 30 minutes per day. These findings are consistent with most studies on the challenges of providing adequate practice in prone play (Bartlett \& Fanning, 2003; Dudek-Shriber \& Zelazny, 2007; Hunter \& Malloy, 2002; Zachry \& Kitzmann, 2011). Expecting parents to implement at least 30 minutes of prone play per day starting on the first day of intervention might have been an impractical recommendation, especially for infants who could not tolerate five consecutive minutes of prone play at baseline. In the future, we will consider making a staged goal for increasing prone time consistent with the infant's gradually improving prone tolerance. For example, 
15 minutes in week 1, 20 minutes in week 2, and 30 minutes in week 3 . Despite the struggle to achieve the "30 minutes" dose, parents in both the groups incorporated prone play in their infant's routine on almost all days they completed the log during the study and demonstrated an increase in the total time spent in prone each week (Table 2). In the future, the research team will continue to include weekly followups (day 7 and day 14) with the parents as a method to keep parents engaged in the intervention, foster their understanding on prone play, and provide ongoing suggestions for implementation. Overall, our results highlight that even highly motivated parents who agreed to participate in a study of prone play have difficulty with implementing at-home interventional strategies with weekly reinforcement.

Compliance with the use of the PPAC was limited to only $25 \%$ of parents reporting $>30$ minutes of prone play with the PPAC per day. All parents were able to verbalize and set the "just right" levels of prone play as recommended. However, only one parent implemented her knowledge of incrementing the threshold heights according to the intervention protocol. Other parents were eager to advance the intensity at a quicker rate, raising the questions of if a higher intensity and shorter dose or lower intensity and a higher dose would be more effective and appealing to the parents. These questions cannot be answered by this study, but could be in future research. While not compliant with the recommended intervention progression, use of the PPAC was welcomed by parents. They saw the new technology as a way to support their infant's development. Thus, the intervention protocol may need to be updated for future research, but its use was feasible and acceptable to parents.

Parents often reported that the paper version of the activity log was too long and even with the electronic version they sometimes missed the notifications to complete the activity log. In future studies, the PPAC should be modified to add the capacity to store data that could be used to track the amount of time the PPAC was used, number of times the infant raised the head above the threshold, and information on the activity of infants in the PPAC. In addition to helping researchers, this type of data could provide positive reinforcement to parents to increase the use of the device each day or for parents to monitor their infant's progress.

\section{Outcome Measures Feasibility}

Both the measures of motor development (AIMS and GMFM-88) and the prone tolerance measure developed for this study are feasible to administer in infants with poor prone tolerance. The prone tolerance measure developed for this study is a feasible and reliable measure. It can quantify change in prone tolerance of infants in both the groups. Parents identified poor prone tolerance in 76\% of 3-to 6-month-old infants. These infants also demonstrated poor prone tolerance on the newly developed measure of prone tolerance. This finding is consistent with past reports that parents can identify their infant's poor tolerance for prone play (Zachry \& Kitzmann, 2011). Also, all infants in the study cooperated during the assessment visits, making it possible for the research team to complete data collection in a single visit.

\section{Pilot Efficacy Study Guidelines}

While the purpose of this study was not to evaluate efficacy of the interventions, the increase in prone tolerance and motor development appears to be present in infants 
whose parents also reported an increase in the duration of prone play at home. The two infants (infant 2 and infant 3 in Table 2) with the greatest increase in prone tolerance also were reported to spend $>30$ minutes in prone at home (in and out of PPAC). These infants also had a higher increase in the motor skills on the GMFM88 compared to the other infants in the study. It is also important to put on record the performance of infant 8 , who had the highest motor scores at baseline compared to other infants and increased her prone tolerance similar to infant 2 and 3. Since infant 8's parent did not fill out the activity log after week 2 of the study, we could not assess the quality of prone play this particular infant might have received at home throughout the study. On the whole, infants in the Reward contingency group had a higher change in their prone tolerance and motor development in a span of three weeks compared to infants in the Education group. The large effect size seen in the prone tolerance score and motor skills from baseline to end of the intervention period reflected the change in motor development and prone tolerance seen in infants in the Reward contingency group. Although, considering the feasibility nature of this study, conclusions cannot be drawn on the efficacy of the proposed intervention to improve motor development and prone tolerance, the inter-relationship between these outcome measures in this study suggests the need to retain the measures in future research.

As we explored the potential factors, which may influence the efficacy of the intervention, we did not see infants' age, motor skills, and prone tolerance at baseline influence infants' motor skills and prone tolerance (Table 2). However, infants who practiced $>30$ minutes of prone play at home had the maximum gain in prone tolerance and a measurable change seen in their motor skills (Table 2). Future studies should continue to measure the daily duration of any prone play to allow for quantification of the changing prone opportunities that may occur in response to the intervention. Although we did not have a direct measure of parents' perceptions of the use of PPAC in their infant's play routine, we did transcribe parents' spontaneous comments about the device during the therapist sessions. After using the device for a week, a parent (infant 2) stated, "So yeah, the device isn't too difficult to use"; and "He's uh actually had a few sessions where he's staying on his stomach without fussing for five minutes, which was Christmas miracle". Another parent (infant 4) stated, "Sometimes she'll do little dances in the gym (PPAC)". The positive feedback provided by parents on the utility of the PPAC is encouraging for the research team to assess the efficacy of an intervention based on reward contingency to improve prone tolerance and motor development in infants in the next study.

\section{Limitations}

There are some important limitations to the current study. The first is the phrasing of the options ( $<15$ minutes, $15-30$ minutes, and $>30$ minutes) parents had in the activity $\log$ to report the amount of time their infant spent in prone. We asked parents to incorporate at least 30 minutes of prone play per day for 15 days over three weeks, but the activity log did not have an option of "30 minutes" for parents to check off. In addition, the 15-30 minutes range may not be sensitive enough to capture small increments of change. As such, we decided to evaluate the "> $>30$ minutes" option to assess parent's adherence to follow the recommended dose. So, if a parent reported 15-30 minutes because the infant exactly practiced the 
recommended 30 minutes, the infant was reported as not meeting the recommended amount based on the log data. This error should be rectified in the future as it might have led to underrepresentation of parents' ability to follow the dose of the interventions. Second, we do acknowledge that although a blinded researcher scored the motor development measures (AIMS and GMFM-88), these measures were administered by an unblinded researcher and may have caused a bias in the motor scores. The future efficacy trial should follow the best research practice of having a blinded researcher administer and score the primary outcome, which is the change in motor development scores, as this will determine the efficacy of the proposed intervention.

\section{Conclusion}

Our study demonstrated the feasibility of the proposed intervention and provided us with valuable suggestions for some modifications. A large scale randomized clinical trial is needed to determine whether an intervention based on reward contingency has the potential to increase prone play and improve motor development. This intervention can benefit full-term infants with poor prone tolerance and low prone motor skills as well as those at the risk of developmental delays.

\section{Acknowledgments}

The authors thank the parents and dedicated staff of the Motor Development Lab at Virginia Commonwealth University (VCU) for their time and efforts in this study. The authors are grateful to the VCU, Graduate School Dissertation Assistantship award for funding Dr. Tripathi in the final year of her doctoral studies. The authors appreciate Dr. Sonia Khurana, Ms. Gullnar Syed, Ms. Kayla Brennan, Ms. Katherine Greenberg, and Ms. Lauren Janelle Libiran for their many hours of assisting in data collection and behavioral coding for this study. The authors also thank Dr. Tripathi's dissertation committee members for their guidance in the planning and dissemination of the study.

\section{References}

Back to sleep, tummy to play. (2017, January 20). Retrieved from https://www .healthychildren.org/English/ages-stages/baby/sleep/Pages/Back-to-Sleep-Tummy-toPlay.aspx

Bartlett, D.J., \& Fanning, J.E.K. (2003). Relationships of equipment use and play positions to motor development at eight months corrected age of infants born preterm. Pediatric Physical Therapy, 15(1), 8-15. PubMed ID: 17057426 doi:10.1097/01.PEP .0000051693 .49199 .41

Brazelton, T.B., \& Nugent, J.K. (1995). Neonatal behavioral assessment scale (3rd ed.). London, UK: Mac Keith Press.

Chizawsky, L.L., \& Scott-Findlay, S. (2005). Tummy Time!. Nursing for Women's Health, 9(5), 382-387. PubMed ID: 32449377

Collett, B., Breiger, D., King, D., Cunningham, M., \& Speltz, M. (2005). Neurodevelopmental implications of "deformational" plagiocephaly. Journal of Developmental Behavioral Pediatrics, 26(5), 379-389. PubMed ID: 16222180 doi:10.1097/ 00004703-200510000-00008 
Darrah, J., Bartlett, D., Maguire, T.O., Avison, W.R., \& Lacaze-Masmonteil, T. (2014). Have infant gross motor abilities changed in 20 years? A re-evaluation of the Alberta Infant Motor Scale normative values. Developmental Medicine \& Child Neurology, 56(9), 877-881. PubMed ID: 24684556 doi:10.1111/dmcn.12452

Dudek-Shriber, L., \& Zelazny, S. (2007). The effects of prone positioning on the quality and acquisition of developmental milestones in four-month-old infants. Pediatric Physical Therapy, 19(1), 48-55. PubMed ID: 17304097 doi:10.1097/01.pep.0000234963 .72945.b1

Dunst, C.J., Trivette, C.M., Raab, M., \& Masiello, T.L. (2008). Early child contingency learning and detection: Research evidence and implications for practice. Exceptionality, 16(1), 4-17. doi:10.1080/09362830701796743

Dusing, S.C., Harbourne, R.T., Lobo, M.A., Westcott-McCoy, S., Bovaird, J.A., Kane, A.E., . . Brown, S.E. (2019). A physical therapy intervention to advance cognitive and motor skills: A single subject study of a young child with cerebral palsy. Pediatric Physical Therapy, 31(4), 347-352. PubMed ID: 31568380 doi:10.1097/PEP .0000000000000635

Dusing, S.C., Tripathi, T., Marcinowski, E.C., Thacker, L.R., Brown, L.F., \& HendricksMuñoz, K.D. (2018). Supporting play exploration and early developmental intervention versus usual care to enhance development outcomes during the transition from the neonatal intensive care unit to home: A pilot randomized controlled trial. $B M C$ Pediatrics, 18(1), 46. PubMed ID: 29426320 doi:10.1186/s12887-018-1011-4

Eldridge, S.M., Chan, C.L., Campbell, M.J., Bond, C.M., Hopewell, S., Thabane, L., \& Lancaster, G.A. (2016). CONSORT 2010 statement: Extension to randomised pilot and feasibility trials. Pilot and Feasibility Studies, 2(1), 64. doi:10.1186/s40814-0160105-8

Enright, M.K., Rovee-Collier, C.K., Fagen, J.W., \& Caniglia, K. (1983). The effects of distributed training on retention of operant conditioning in human infants. Journal of Experimental Child Psychology, 36(2), 209-225. PubMed ID: 6631328 doi:10.1016/ 0022-0965(83)90030-9

Gerhardstein, P., Kraebel, K., \& Tse, J. (2006). Using operant techniques with humans infants. The Behavior Analyst Today, 7(1), 56-69. doi:10.1037/h0100142

Griffith, H., \& Biswas, S. (2017, July). Home-based upper extremity rehabilitation support using a contactless ultrasonic sensor. In 2017 39th Annual International Conference of the IEEE Engineering in Medicine and Biology Society (EMBC) (pp. 853-856). IEEE.

Harbourne, R.T., Dusing, S.C., Lobo, M.A., Westcott-McCoy, S., Bovaird, J., Sheridan, S., ... Marcinowski, E.C. (2018). Sitting together and reaching to play (START-play): Protocol for a multisite randomized controlled efficacy trial on intervention for infants with Neuromotor disorders. Physical Therapy, 98(6), 494-502. PubMed ID: 29767802 doi:10.1093/ptj/pzy033

Heathcock, J.C., Bhat, A.N., Lobo, M.A., \& Galloway, J. (2004). The performance of infants born preterm and full-term in the mobile paradigm: Learning and memory. Physical Therapy, 84(9), 808-821. PubMed ID: 15330694 doi:10.1093/ptj/84.9.808

Hewitt, L., Stanley, R.M., \& Okely, A.D. (2017). Correlates of tummy time in infants aged 0-12 months old: A systematic review. Infant Behavior and Development, 49, 310-321. PubMed ID: 29096238 doi:10.1016/j.infbeh.2017.10.001

Hunter, J.G., \& Malloy, M.H. (2002). Effect of sleep and play positions on infant development: Reconciling developmental concerns with SIDS prevention. Newborn and Infant Nursing Reviews, 2(1), 9-16. doi:10.1053/nbin.2002.31484

Klimo, P., Jr., Lingo, P.R., Baird, L.C., Bauer, D.F., Beier, A., Durham, S., . . Tamber, M.S. (2016). Congress of Neurological Surgeons systematic review and evidencebased guideline on the management of patients with positional plagiocephaly: The role of repositioning. Neurosurgery, 79(5), E627-E629. 
Koo, T.K., \& Li, M.Y. (2016). A guideline of selecting and reporting intraclass correlation coefficients for reliability research. Journal of Chiropractic Medicine, 15(2), 155-163. PubMed ID: 27330520 doi:10.1016/j.jcm.2016.02.012

Koren, A., Reece, S.M., Kahn-D'angelo, L., \& Medeiros, D. (2010). Parental information and behaviors and provider practices related to tummy time and back to sleep. Journal of Pediatric Health Care, 24(4), 222-230. PubMed ID: 20620848 doi:10.1016/j.pedhc .2009.05.002

Morgan, C., Darrah, J., Gordon, A.M., Harbourne, R., Spittle, A., Johnson, R., \& Fetters, L. (2016). Effectiveness of motor interventions in infants with cerebral palsy: A systematic review. Developmental Medicine \& Child Neurology, 58(9), 900-909. PubMed ID: 27027732 doi:10.1111/dmcn.13105

Novak, I., Mcintyre, S., Morgan, C., Campbell, L., Dark, L., Morton, N., ... Goldsmith, S. (2013). A systematic review of interventions for children with cerebral palsy: State of the evidence. Developmental Medicine \& Child Neurology, 55(10), 885-910. PubMed ID: 23962350 doi: $10.1111 / \mathrm{dmcn} .12246$

Piper, M.C., Pinnell, L.E., Darrah, J., Maguire, T., \& Byrne, P.J. (1992). Construction and validation of the Alberta Infant Motor Scale (AIMS). Canadian Journal of Public Health, 83(Suppl. 2), S46-S50. PubMed ID: 1468050

Rovee-Collier, C.K., \& Gekoski, M.J. (1979). The economics of infancy: A review of conjugate reinforcement. Advances in Child Development and Behavior, 13, 195-255. PubMed ID: 484323

Russell, D., Rosenbaum, P., Wright, M., \& Avery, L.M. (2013). Gross motor function measure (GMFM-66 \& GMFM-88) user's manual (2nd ed.). London, UK: Mac Keith Press.

Sargent, B., Reimann, H., Kubo, M., \& Fetters, L. (2015). Quantifying learning in young infants: Tracking leg actions during a discovery-learning task. Journal of Visualized Experiments, (100), e52841. doi:10.3791/52841

Scott, T.M. (2001). A schoolwide example of positive behavioral support. Journal of Positive Behavior Interventions, 3(2), 88-94.

Thelen, E. (1995). Motor development: A new synthesis. American Psychologist, 50(2), 79-95. PubMed ID: 7879990 doi:10.1037/0003-066X.50.2.79

Thorne, S., \& Kamps, D. (2008). The effects of a group contingency intervention on academic engagement and problem behavior of at-risk students. Behavior Analysis in Practice, 1(2), 12-18.

Tripathi, T., Dusing, S., Pidcoe, P.E., Xu, Y., Shall, M.S., \& Riddle, D.L. (2019). A motor learning paradigm combining technology and associative learning to assess prone motor learning in infants. Physical Therapy, 99(6), 807-816. PubMed ID: 31155666 doi:10.1093/ptj/pzz066

Van Vlimmeren, L.A., van der Graaf, Y., Boere-Boonekamp, M.M., L'Hoir, M.P., Helders, P.J., \& Engelbert, R.H. (2007). Risk factors for deformational plagiocephaly at birth and at 7 weeks of age: A prospective cohort study. Pediatrics, 119(2), e408-e418. PubMed ID: 17272603 doi:10.1542/peds.2006-2012

Vygotsky, L.S. (1978). Mind in society: The development of higher psychological processes. Cambridge, MA: Harvard University Press.

Zachry, A.H., \& Kitzmann, K.M. (2011). Caregiver awareness of prone play recommendations. American Journal of Occupational Therapy, 65(1), 101-105. PubMed ID: 21309377 doi:10.5014/ajot.2011.09100 